\title{
Review of: "A brief introduction to the COM-B Model of behaviour and the PRIME Theory of motivation"
}

\author{
Luke Davis ${ }^{1}$ \\ 1 Yale University
}

Potential competing interests: The author(s) declared that no potential competing interests exist.

This is an excellent precis of two tools that I have found powerful as an epidemiologist working in the design, implementation, and evaluation of complex interventions in low- and middle-income countries. These theories lies at the heart of a large project led by Michie and West to develop universal, evidence-informed tools for understanding and influencing human behavior within the multi-level context of the environment and systems, with the goal of improving health and well-being. Motivation is at the heart of the COM-B system and the PRIME theory explains the complexities of motivation in ways that health researchers without deep grounding in psychological theory can understand. With the link to the larger Behaviour Change Wheel, COM-B in turn provides the grounding for developing interventions and policies to influence change within complex (i.e. multi-component) implementation strategies. We have found that these theories are also very practical for planning implementation projects and can be widely taught and applied by implementation teams, and an extensive literature now documents the applicability of COM-B to real-world problems. 